\title{
Development of Healthcare Kiosk for Checking Heart Health
}

\author{
Riyanto Sigit, Zainal Arief, Mochamad Mobed Bachtiar \\ Politeknik Elektronika Negeri Surabaya \\ Kampus PENS, Jalan Raya ITS Sukolilo, Surabaya 60111 \\ Tel: (031) 594 7280; Fax: (031) 5946114 \\ E-mail: riyanto@pens.ac.id,zar@pens.ac.id,mobed@pens.ac.id
}

\begin{abstract}
The main problem encountered nowadays in the health field, especially in health care is the growing number of population and the decreasing health facilities. In this regard, healthcare kiosk is used as an alternative to the health care facilities. Heart disease is a dangerous one which could threaten human life. Many people have died due to heart disease and the surgery itself is still very expensive. To analyze heart diseases, doctor usually takes a video of the heart movement using ultrasound equipment to distinguish between normal and abnormal case. The results of analysis vary depending on the accuracy and experience of each doctor so it is difficult to determine the actual situation. Therefore, a method using healthcare kiosk to check the heart health is needed to help doctor and improve the health care facilities.

The aim of this research is to develop healthcare kiosk which can be used to check the heart health. This research method is divided into three main parts: firstly, preprocessing to clarify the quality of the image.In this section, the writers propose a Median High Boost Filter method which is a combined method of Median Filtering and High Boost Filtering. Secondly, segmentation is used to obtain local cavities of the heart. In this part, the writers propose using Triangle Equation that is a new method to be developed. Thirdly, classification using Partial Monte Carlo method and artificial neural network method; these methods are used to measure the area of the heart cavity and discover the possibility of cardiac abnormalities. Methods for detecting heart health are placed in the kiosk. Therefore, it is expected to facilitate and improve the healthcare facilities.
\end{abstract}

Keywords: Healthcare kiosk, heart health, reprocessing, segmentation, classification.

\section{INTRODUCTION}

Heart disease is very dangerous and it causes the death of many people throughout the world. Clinics have focused their research on diagnosing cardiac disease. Cardiac function can be measured using ultrasound, Magnetic Resonance Imaging (MRI), Computed Tomography (CT), X-Ray, 
Positron Emission Tomography (PET) and Single Photon Emission Tomography (SPECT).

This study used ultrasound and echocardiography to measure cardiac function. Echocardiography is advantageous because it is affordable, widely used, provides a great amount of information, non-invasive, does not expose the patient to radiation and it can be applied to patients in critical condition. The results are directly evident. Standard images used in echocardiography are the short-axis, long-axis, two-chamber and four-chamber views. At present, the reading of echocardiograms by experts is conducted using standard clinical practice. However, this task requires accuracy and experience in diagnosing the heart cavity structure.

Various studies have focused on segmenting cardiac cavities [1, 2]. However, there is still room for the innovation and development of new methods and algorithms for further improvement. To perform segmentation and detection of echocardiographic images, a number of researchers have used short-axis images [3]. Klingler et al. [4], Lacerda et al. [5] and dos Reis et al. [6] used semi-automatic detection methods, whereas Hae-Yeoun et al. [3] and Ohyama et al. [7] developed fully automated detection methods. Works on using snake's algorithm to perform segmentation of cardiac cavity are reported by Jierong, et al [8], Moursi\&El-Sakka[9] and Ranganath[10].

In this study, to develop kiosk for checking heart health with an alternative and implementation the simpler method called triangle method is proposed to delineate the boundary of cardiac cavity from echocardiographic images. In order to obtain an initial estimate of the boundary, combinations of filtering and morphological operations are applied to the original image. The filtering process involves application of high boost filter along with morphological and thresholding operators to eliminate noise and convert the image into a binary image. The use of a high boost filter is meant to enhance the high frequency components without affecting the low frequency components. This filter is followed by a negative Laplacian filter for edge detection. A region filter is then applied to confine the boundary detection region. The triangle method is then used to reconstruct a more precise border by pruning and connecting the unwanted sections of the boundary and lines. All the method will be implemented in the healthcare kiosk.

\section{RELATED WORKS}

The main objective of this work is to development of healthcare kiosk for checking hearth health. In [1], Noble and Boukerroui survey several methods and techniques for ultrasound segmentation. Petitjean and Dacher[2] review segmentation methods in short axis cardiac MRI. Lee et al. [3] used automatic segmentation for cardiac images using iterative thresholding and an active contour model. Klingler et al. [4] applied mathematical morphology to perform the segmentation of echocardiography images. Lacerda et al. [5] presented radial search for the segmentation of cardiac cavities. Chalana et al. [11] used multiple active contour models for 
cardiac boundary detection. Laine and Zong[12] applied border identification that depended on the shape modeling and border reconstruction from a set of images. Ohyama et al. [7] presented a ternary threshold method for detection of the left ventricular endocardium. Maria et al. [6] used semiautomatic detection of the left ventricular border. Sigit et al. [13] applied the triangle equation for automatic segmentation of the cardiac cavity, but only worked for cases where there are only open major boundary without isolated minor closed contours. In this paper, improvement of this method is presented that is able to handle more general cases.

Segmentation using snakes have been conducted by several researchers. Kass et al. [14] was the first to introduce the snake algorithm. Chenyang and Prince[15] developed a new model of external forces for snake, called gradient vector flow (GVF). Jierong et al. [8] used morphological operations and the snake model to detect the boundary of echocardiographic images. Moursi and El-Sakka[9] presented an efficient algorithm for determining a good initial centroid contour in ultrasound images with the snake algorithm. Ranganath[10] developed algorithms based on snakes for extracting blood pool contours from cardiac MRI. Itai et al. [16] used automatic detection of abnormal shadow by using snake for segmentation of lung area. Jaffar et al. [17] describe a method for full automatic segmentation based on Fuzzy entropy and morphological image processing techniques from CT-Scan images.Sigit et al. [18] proposed a method for segmentation of cardiac cavity image using search contour and snakes.

The snake is an active contour model. Snake can be used to find the boundary of an object in an image starting from an initial boundary estimate. It searches boundary images by minimizing the energy function. The total energy of the snake is defined as in (1).

$$
E=\frac{1}{2} \int\left(\alpha\left|v^{\prime}(s)\right|^{2}+\beta\left|v^{\prime \prime}(s)\right|^{2}-\lambda|v(s)| \mid d s\right.
$$

The weakness of the snake algorithm is that it is dependent on the initial boundary estimate and setting of energy function variables $(\alpha, \beta, \lambda)$. In this study, we propose an algorithm called triangle method combined with filtering and a morphology operation that is independent of initial estimate and does not require any variable to be set.

\section{ORIGINALITY}

In this paper, development of healthcare kiosk for checking hearth heath is proposed using triangle method.The main task of the proposed triangle method is to prune and connect these boundaries to form a single closed boundary to delineate the cardiac cavity. This is achieved by successively scanning the points on noisy boundary using the corners of a triangle subtended from the center of the initial boundary.

Triangle method has been successful gives better results compared with the snake algorithm. The proposed method can provide solutions of 
image segmentation for cardiac cavity images. The triangle method for image segmentation is a novelty and not used mainly for segmentation.Results from test using actual echocardiographic images show that the proposed method give better accuracy compared to snake algorithm. The proposed method is also easier to use as it does not need any tuning parameter.

\section{SYSTEM DESIGN}

The preprocessing steps involves several filtering processes such as high boost, morphological, thresholding, negative Laplacian and region filtering as shown in Figure 1.

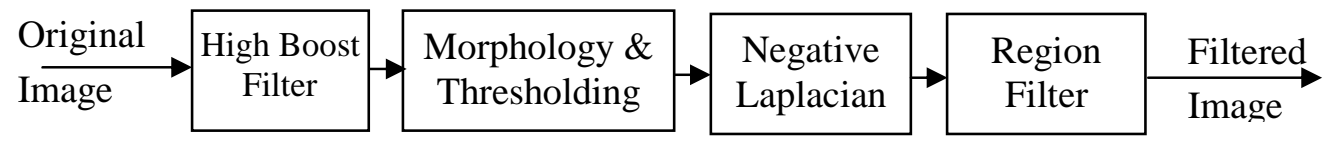

Figure 1. Block diagram of the pre-processing steps

The procedure begins by reading the input file, i.e., the AVI video files comprising the stored ultrasound images of the short-axis display of the cardiac cavity. The input file is read by view image, which then converts the video recording into a sequence of image frames to enable the images to be processed and analyzed.

Next, a high boost filter is implemented to enhance the image because the images obtained via view image are not clear. By using the high boost filter, the image can be improved, and the cardiac cavity can be made more prominent because in high boost filtering, the high frequency components of the image are enhanced, while the low frequency components remain mostly unchanged.

Though the image is somewhat improved, there is still noise due to the enhancement of the high frequency components. To suppress this noise, a morphological technique using the open and close operators is used to remove speckle noise and sharpen the edges of the cavity, which is followed by thresholding to convert the image into a binary format. Next, the binary image is subjected to a negative Laplacian filter to determine the boundary of the cavity. The implementation of a negative Laplacian filter involves taking derivatives and performing convolution on the binary image to extract the high-pass information directly. By doing so, a sharpened image can be obtained, and the edges of the cavity can be clearly determined. Next, the image is further subjected to the region filtering technique to eliminate small and insignificant contours. Before region filtering, the center point and radius of the circles are preset. After region filtering, the boundary of the cardiac cavity in the image is almost evident but still contaminated with other contours. To completely remove these other unwanted contours and maintain only the desired contour of the cardiac cavity, a method based on intersecting connecting line is used. The use of the intersecting connecting was reported and described in [13]. 


\subsection{Cardiac Image View}

Typically, cardiac cavity images are presented using standard viewing methods, such as the short-axis and long-axis views. In this research, shortaxis images are processed and analyzed to test the proposed segmentation and detection algorithm using the triangle equations.

The procedure starts by reading the input file, i.e., the AVI video files comprising the stored ultrasound images of the short-axis display of the cardiac cavity. The input file is read using view image, which then converts the video recording into a sequence of image frames to allow the images to be processed and analyzed.

\subsection{High Boost Filter}

The high boost filter is implemented first. Itis one of the sharpening operators in image processing. A high pass filter reduces the original image using a low pass image, asshown in (2).

High pass $=$ Original - Low pass

A high boost filter multiplies the original image by a factor $A$, and the result is then reduced by the low pass image, asshown in (3).

High boost $=\mathrm{A} *$ Original - Low pass

Alternatively, the original image can be multiplied by (A-1), and the result can be added to the high pass image, as shown in (4). In the case where $A$ is equal to1, it is called a high pass filter, but if $A$ is greater than 1 , it is called a high boost filter.

$$
\begin{aligned}
& \text { High boost }=(A-1) * \text { Original }+ \text { Original }- \text { Low pass } \\
& \text { High boost }=(A-1) * \text { Original }+ \text { High pass }
\end{aligned}
$$

The high-boost filter is used to enhance high frequency component while maintaining the low frequency components. The kernel from the high boost filter is shown in (5):

$$
\mathrm{W}_{\text {highboost }}=(\mathrm{A}-1) * \mathrm{~W}_{\text {original }}+\mathrm{W}_{\text {highpass }}
$$

For example, $W_{\text {highboost }}=(A-1) *\left[\begin{array}{ccc}0 & 0 & 0 \\ 0 & 1 & 0 \\ 0 & 0 & 0\end{array}\right]+\left[\begin{array}{ccc}0 & -1 & 0 \\ -1 & 4 & -1 \\ 0 & -1 & 0\end{array}\right]$

$$
W_{\text {highboost }}=(A-1) *\left[\begin{array}{ccc}
0 & 0 & 0 \\
0 & 1 & 0 \\
0 & 0 & 0
\end{array}\right]+\left[\begin{array}{ccc}
-1 & -1 & -1 \\
-1 & 8 & -1 \\
-1 & -1 & -1
\end{array}\right]
$$


Figure 2 and Figure 3 show the effect of the high pass filter and high boost filter. Figure 2(a) is the short-axis image of the left ventricular cardiac cavity, which was obtained from echocardiography. Figure2(b)is the effect of a high pass filter with central kernels 4. Figure2(c) shows the effect of a high pass filter with the center of kernel8. Figure 3 represents the effects of the high boost filter with $A=2,4,8,16,32$ and 64 . The image becomes sharper as the factor A increases.

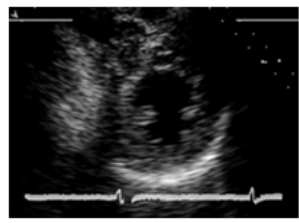

(a)

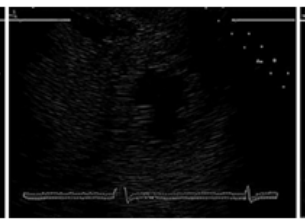

(b)

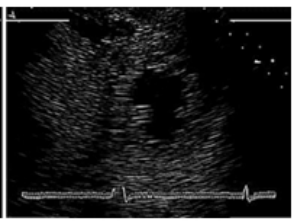

(c)

Figure 2. The effect of the high pass filter. (a) Original short axis image. (b) High pass 4. (c) High pass 8.

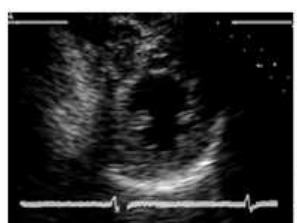

(a)

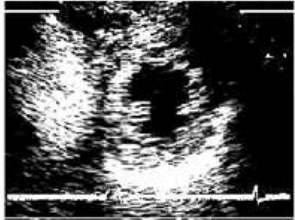

(b)

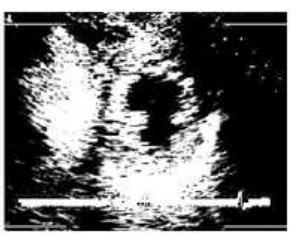

(c)

Figure 3. The effect of the high boost filter. (a) Original short axis image. (b) High pass 4. (c) High pass 8.

Sigit et al. [13] presented high boost filter with uses the spatial mask from Lacerda[5]. Inthisstudy, an Avalue of 32 was used.Figure 4 shows the spatialmaskassociated with this value of A. Using this high boost filter, the image can be enhanced, and the cardiac cavity can be made more visible as shown in Figure 3.

\begin{tabular}{|c|c|c|}
\hline-1 & -1 & -1 \\
\hline-1 & 40 & -1 \\
\hline-1 & -1 & -1 \\
\hline
\end{tabular}

Figure 4. Spatial mask used for the high boost filter

\subsection{Morphology and Thresholding}

After implementing the high boost filter, the morphology operation is used to remove noise. Basic morphology operations are dilation and erosion. Both dilation and erosion are produced by the interaction of a set of structureelements with the image. This structure element has the shape and origin shown in Figure 5(a).

Suppose A is the set of pixels, and B is a structure element. Structure element B dilates set A by filling holes with certain shapes and sizes provided by the structure elements, such as in Figure 5(b). Structure element B erodes 
set A by eliminating certain shapes and sizes of the structure provided by the structure elements, such in as Figure 5(c).

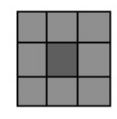

(a)

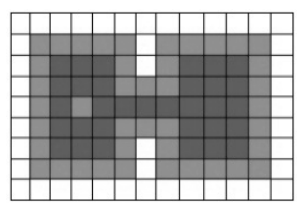

(b)

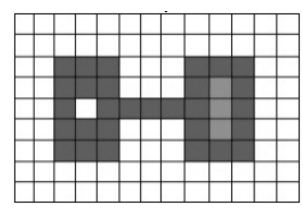

(c)

Figure 5. Morphologyoperation. (a)Structureelements (b)Dilation (c)Erosion

Dilation $A \oplus B$ can be written as in formula (6), while the erosion $A \Theta B$ can be written as in formula (7), where $\hat{B}$ is the reflection of $\mathrm{B}$ followed by a shift z.

$$
\begin{aligned}
& A \oplus B=\left\{z \mid(\hat{B})_{z} \cap A \neq \Phi\right\} \\
& A \Theta B=\left\{z \mid(B)_{z} \subseteq A\right\}
\end{aligned}
$$

This study uses morphological operations with the opening and closing algorithm. The main purpose of the opening and closing algorithm is to reduce speckle noise in the cardiac cavity image. The opening algorithm involves eroding image A by B and dilating it by B. Mathematically, it is achieved by using the mathematical notation of the opening algorithm shown in Equation 8. The closing algorithm is implemented by dilating image A and eroding it by $\mathrm{B}$. The mathematical notation of the closing algorithm is shown in Equation 9.

$$
\begin{aligned}
& A \circ B=(A \Theta B) \oplus B \\
& A \bullet B=(A \oplus B) \Theta B
\end{aligned}
$$

Figure6 illustrates the effect ofmorphology using the structure elements circle, crossandellipse. In this study, an elliptical structure element with a $3 \times 3$ matrix structure was used because the shape and number of these structures was found to give the best result.

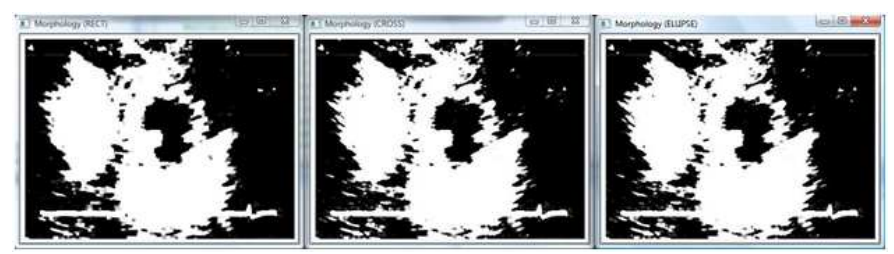

Figure 6. The effect ofmorphology by using the structure lements circle, cross and ellipse 


\subsection{Negative Laplacian Filter}

Next, the negative Laplacian filter, which is a second derivative filter, was realized to find areas of rapid change in the image (i.e., edges). Image edges canbe obtainedin various ways, but the two most commonly used methods for finding the edge of the image a research-based and zerocrossing-based methods. The former method findsthe maximum and minimum from the image edge based on the first derivative, and this method is also called the gradient method. The latter method finds the image edge using the second derivative, and this method is also known as the Laplacian. Figure7 shows the filtered images using various edge detection gradient methods.

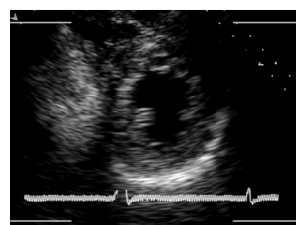

(a)

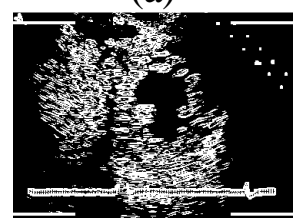

(d)

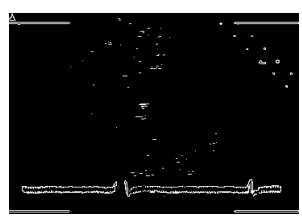

(b)

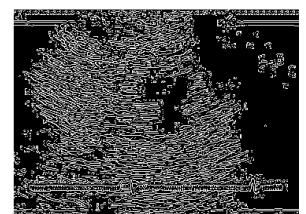

(e)

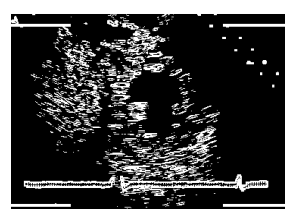

(c)

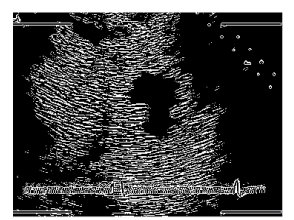

(f)

Figure 7. Filtered images using different edge detection methods. (a) Original image. (b) Robert. (c) Prewitt. (d) Sobel. (e) Canny. (f) negative Laplacian.

It appears that the Canny and negative Laplacian methods give good results but differ in terms of computational speed. Inthis paper, the negative Laplacian for edge detection process was used because it is faster than the Canny method. The Canny method requires a longer processing time than the Laplacian method because it requires the use of the Gaussian derivative and two threshold values to detect both strong and weak edges. There are several ways to find an approximate discrete convolution kernel that approximate the effect of the Laplacian. A possible kernel is shown in Figure 8 below:

\begin{tabular}{|c|c|c|}
\hline 0 & 1 & 0 \\
\hline 1 & -4 & 1 \\
\hline 0 & 1 & 0 \\
\hline
\end{tabular}

Figure 8. Kernel used for negative Laplacian 


\subsection{Region Filter}

Subsequently, the region filter was used to eliminate small contours. The region filter scans the contour and calculates the area of each contour. Regions with an area that is smaller than the pre-determined threshold are eliminated from the contour [5]. The threshold value was set to 25 pixels and was empirically determined. Figure 9 (b) shows the effect of applying region filter to the image given in Figure 9(a).

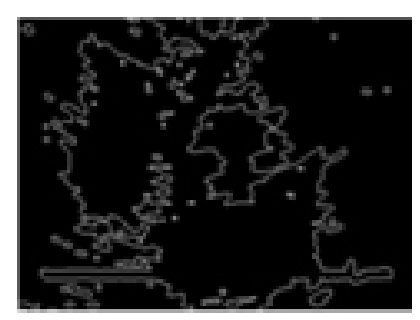

(a)

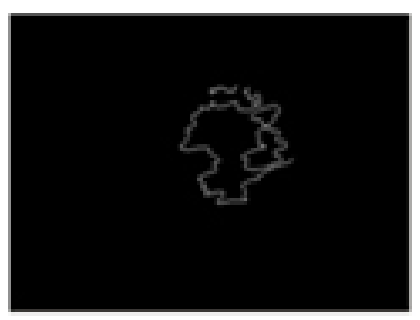

(b)

Figure 9. (a) Imaged formed after high boost filtering and morphology operation.

(b) Image after region filtering.

\subsection{Removing remote contours}

The final process in the pre-processing step is to remove contours that are located far from the major boundary. This is achieved by finding the centroids of all existing contours using Equation 10, as shown below.

$$
\operatorname{centroid}(c)=\left\{\frac{\sum_{k=1}^{n} x k}{n}, \frac{\sum_{k=1}^{n} y k}{n}\right\}
$$

A connecting line is then formed from the center of the boundary to the centroids of each contour. The contour will be maintained if the respective connecting line intersects the inner boundary, and will be removed otherwise. Figure 10 show the image after applying this step.

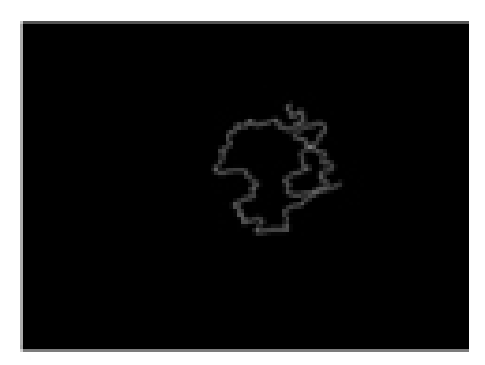

Figure 10. Image after applying intersecting connecting line criteria. 


\subsection{The Triangle Method}

Figure 11(a) shows a triangle, where A, B, and C are the corners and a, $\mathrm{b}$, and $\mathrm{c}$ are the distances between the corners. If $\mathrm{a}, \mathrm{b}$, and $\mathrm{c}$ are known, then the angle of the corner can be calculated using Equations 11 and 12.

$$
\begin{aligned}
& a^{2}=b^{2}+c^{2}-2 b c(\cos A) \\
& A=\operatorname{acos}\left(\left(b^{2}+c^{2}-a^{2}\right) / 2 b c\right)
\end{aligned}
$$

The previous process may produce an image with open and closed boundaries. The boundary is closed when all points are connected, as shown in Figure 11(a). The boundary is open if there are disconnected points or endpoints, as shown in Figure 11(b).

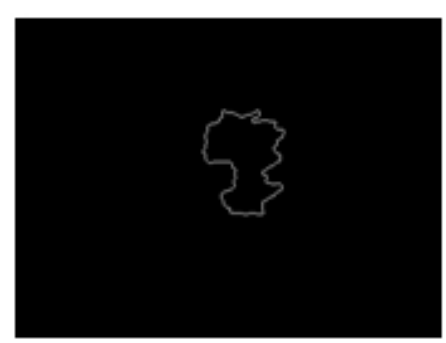

(a)

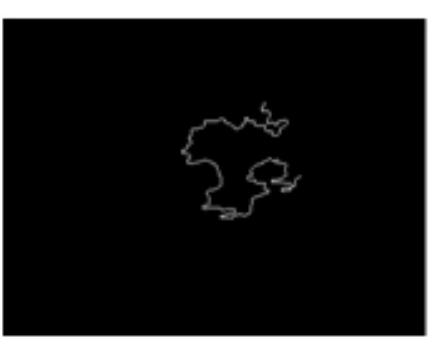

(b)

Figure 11. Boundary of cardiac cavity (a) close boundary (b) open boundary

The boundary of the cardiac cavity can be obtained directly if the contour is closed, but if the boundary of the cardiac cavity is open, it can be obtained by using the triangle method according to the following steps.

1. The first step is to determine the approximate center of the region that seems to be enclosed by the boundary, which can be done by calculating the centroid as in Equation 9. This point is denoted as point A in Figure 12(b).

2. The two endpoints of the boundary are located and are marked as points B and $\mathrm{C}$ in the same figure. The triangle $\mathrm{BAC}$ is formed from these three points with sides $\mathrm{a}, \mathrm{b}$ and $\mathrm{c}$.

3. The gap in the boundary is minimized by first fixing one of the end points, such as point $B$ in Figure 12(c), and allowing point $C$ to transverse inward along the boundary until the point at which the angle BAC is minimized is found. A similar action is then performed for point B to obtain the result shown in Figure 12(d).

4. The open boundary is then closed by connecting a line from point B to point C. 


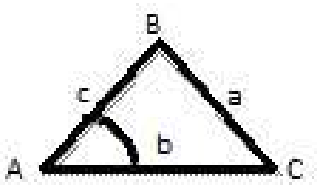

(a)

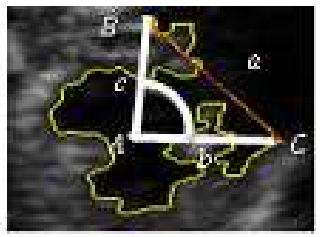

(b)

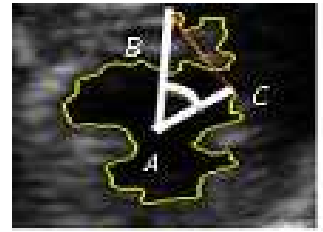

(c)

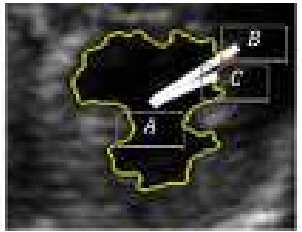

(d)

Figure 12.(a) Triangle sides and angles (b) triangle on the cardiac cavity image. (c) creating a small corner (d) example of finding boundary

The opening gap of the open boundary can be made as small as possible by searching for the two points from each end point such that the angle subtended from these points from the center is minimized. The search starts from one end point of the open boundary and moves inside until it traverses $1 / 3$ of the length of the open boundary. Figure 13(a) shows the variation of the angle of the triangle $\mathrm{BAC}$ with $\mathrm{B}$ fixed and point $\mathrm{C}$ moving inward. The plot shows that the minimum angle is $32.3^{\circ}$ at point 52 from the starting point C. Similarly, with point $C$ fixed at the new location, point B moves inside, and the minimum angle is at point 28, as shown in Figure 13(b)

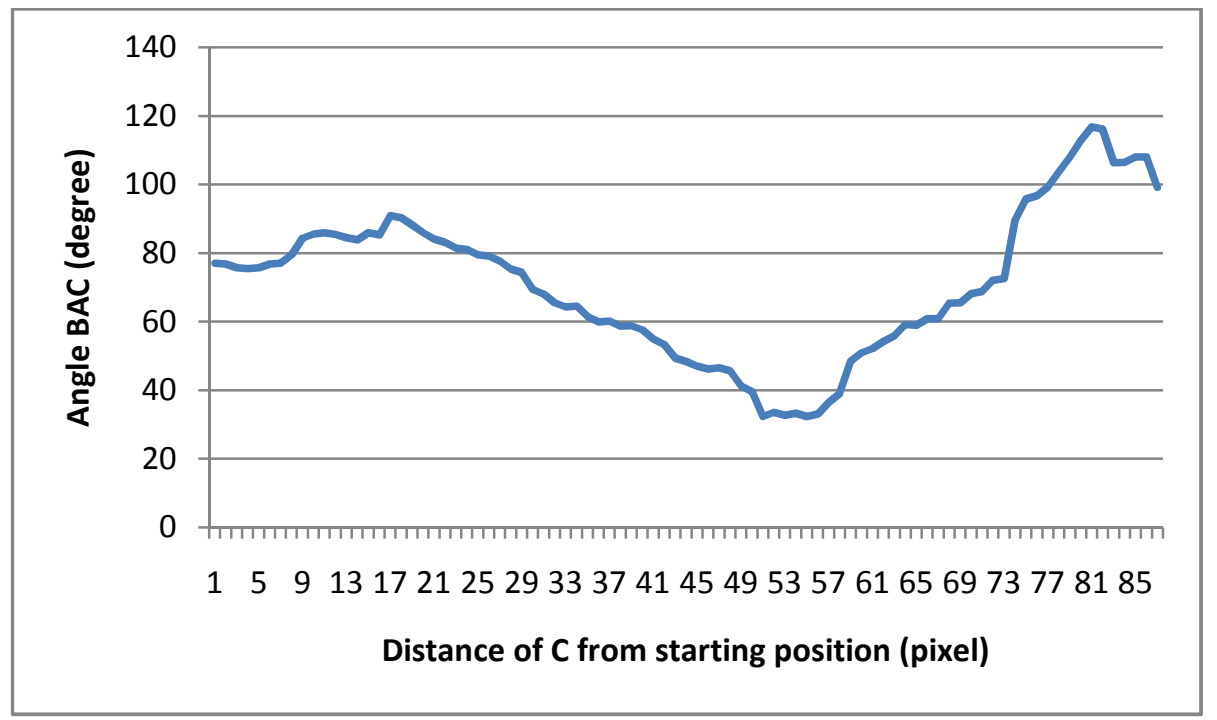

(a) 


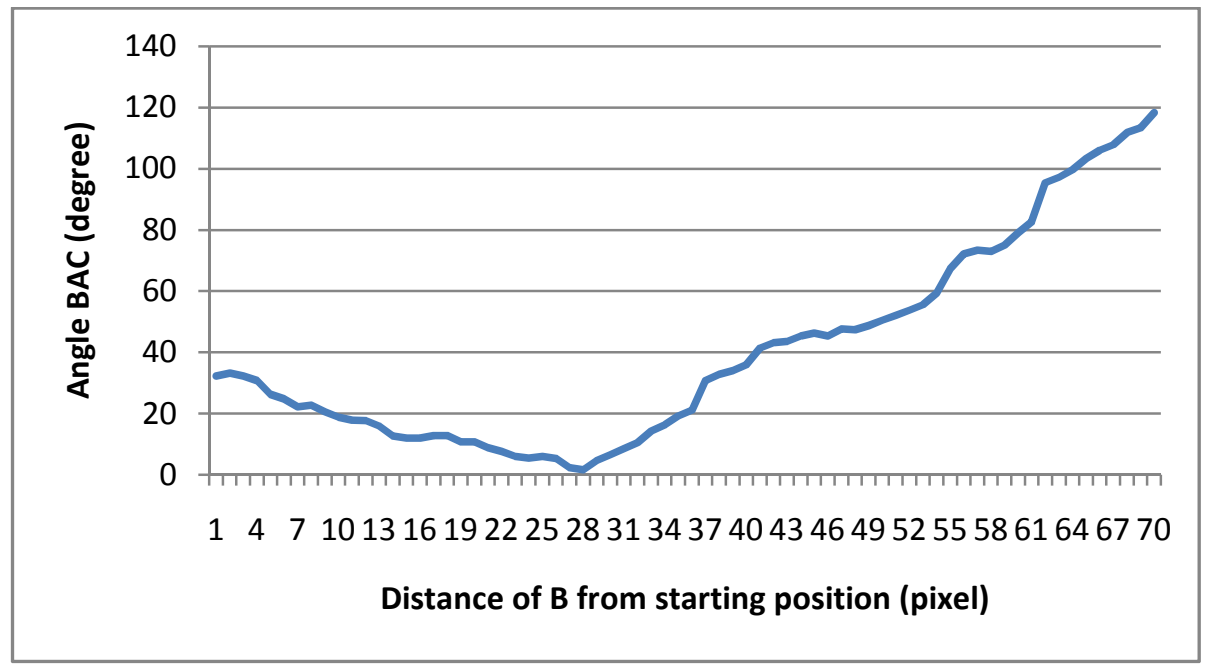

(b)

Figure 13. The angle value is calculated at the two end points. (a) Point $C$ moving inside with point $B$ fixed. (b) Point $B$ moving inside with point $C$ fixed at new location.

There will also be cases when there is an open major boundary with isolated minor closed contours, as shown in Figure 14(a).

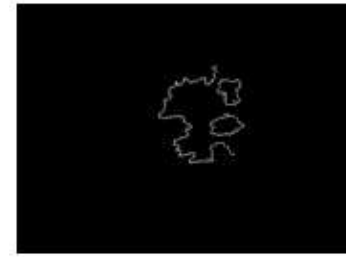

(a)

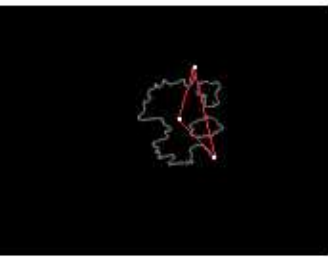

(b)

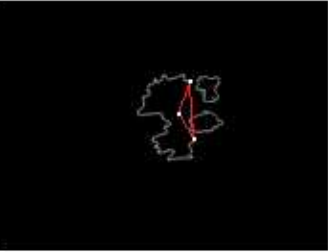

(c)

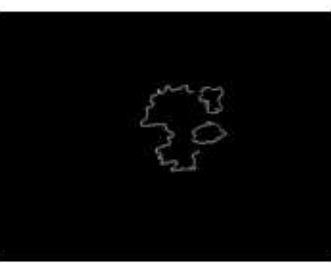

(d)

Figure 14. Using the Triangle Equation method to close the open boundary

For such cases, the following procedure is proposed to form the closed boundary.

1. Minimize the gap in the open boundary using Steps 1-3 in the procedure described earlier. Using Figure 14(a) as an example, the initial triangle was formed, as shown in Figure 14(b). The opening in this boundary is then minimized, as shown in Figure 14(c). The boundary formed after this process is shown in Figure 14(d).

2. The minor closed contours are then merged with the major boundary using the follow steps:

a) Determine the centers of the close contours by calculating the average position of point $\mathrm{x}$ and the average position of point $\mathrm{y}$ on the contour by using Equation 9.

b) Locate the end points of the open boundary. These centers and end points are called the anchor points. Figure 15(a) shows the locations of these 
anchor points.

c) Find the points in the adjacent contours that are closest to the anchor points. These points are called the nearest points.

d) Starting with one of the end points, connect it to the nearest point. From this point, take the path along the contour that is closer to the center of the main boundary until another nearest point is reached. From this point, make a connection to the nearest point in the neighboring contours. The process continues until it is connected to another end point, as shown in Figure 15(b).

e) In the previous step, the sections of the contour between two nearest points were retained as part of the boundary. The other section is then removed to give the final boundary, as shown in Figure 15(c).

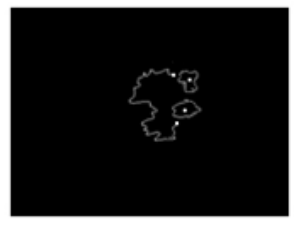

(a)

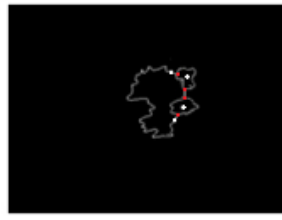

(b)

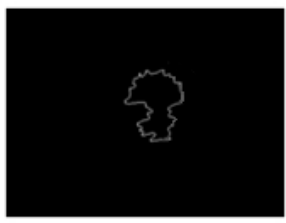

(c)

Figure 15. Constructing the boundary of the cardiac cavity with the open and some closed contours

\section{EXPERIMENT AND ANALYSIS}

The proposed triangle algorithm was tested using a dataset composed of actual ultrasound (US) videos from 109 patients. Seventy-eight videos contained good quality images, and the rest were of poor quality and required additional image enhancement and processing techniques. The testing datasets were extracted from these video recordings, and each comprised 6 to 10 repeated frames. The typical image size was 320 pixels wide and 240 pixels high. Figure 16 shows the delineated cardiac cavity for several video frames from diastole to systole.
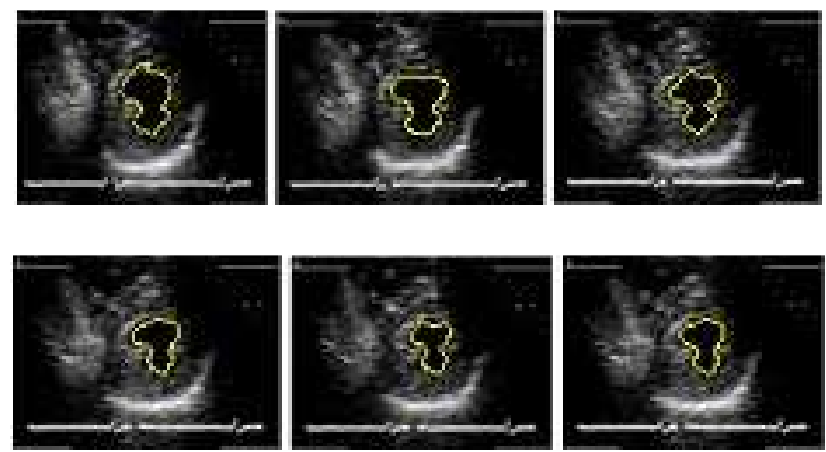

Figure 16. Delineated cardiac cavity during different stages of cardiac cycle

The information about the movement of the boundary of the cardiac cavity provides useful information for the physician on the condition of the myocardium. Useful information that can be extracted from the boundary is 
the area of the cavity that can be used to infer the cardiac pumping performance. In order to assess the performance of the proposed method, the areas of the cardiac cavity in the echocardiographic images from different stages of the cardiac cycle are computed using the triangle method and snake algorithm. These areas are then compared to areas of the cavity delineated manually by the physician. The unit for the area is the number of pixels in the boundary. The comparison is presented in Table 1. From this table, we can see that the areas of the cardiac cavity obtained using the triangle method have an average error of 6.56, compared to an average error of 13.68 based on snake algorithm.

Table 1. Comparison of cardiac cavity areas using triangle and snake

\begin{tabular}{|c|c|c|c|c|c|}
\hline $\begin{array}{c}\text { Images } \\
\text { (Frame) }\end{array}$ & $\begin{array}{c}\text { Area based } \\
\text { on manual }\end{array}$ & $\begin{array}{c}\text { Area based } \\
\text { on snake } \\
\text { algorithm }\end{array}$ & $\begin{array}{c}\text { Area using } \\
\text { triangle } \\
\text { method }\end{array}$ & $\begin{array}{c}\text { Error using } \\
\text { snake } \\
\text { algorithm }\end{array}$ & $\begin{array}{c}\text { Error using } \\
\text { triangle } \\
\text { method }\end{array}$ \\
\hline 1 & 4943 & 5988 & 5329 & 21.14 & 7.81 \\
2 & 4669 & 5735 & 4995 & 22.83 & 6.98 \\
3 & 4481 & 4800 & 4673 & 7.12 & 4.28 \\
4 & 3955 & 4191 & 4038 & 5.97 & 2.10 \\
5 & 3176 & 4059 & 3497 & 27.80 & 10.11 \\
6 & 2731 & 3023 & 2515 & 10.69 & 7.91 \\
7 & 1901 & 2087 & 1817 & 9.78 & 4.42 \\
8 & 2236 & 2104 & 2355 & 5.90 & 5.32 \\
9 & 2013 & 2252 & 2217 & 11.87 & 10.13 \\
\hline \multicolumn{7}{|c|}{ Average error } & 13.68 & 6.56 \\
\hline
\end{tabular}

Figure 17 shows that the calculated areas of the triangle method are almost the same as those computed from the boundary delineated manually by the physician from frame 1 to frame 9.The triangle method clearly shows a better accuracy than the snake algorithm.

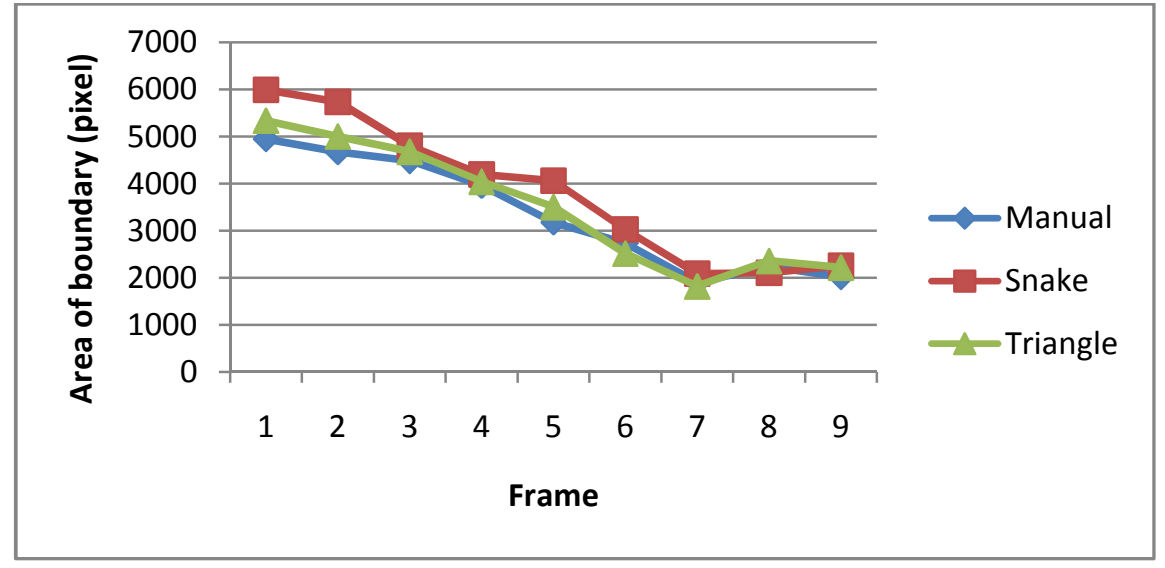

Figure 17. Comparison of cardiac cavity areas using several methods 


\section{CONCLUSION}

The proposed method presents solution for healthcare kiosk for checking heart health. The proposed method can made preprocessing image to be clearly, segmentation can get the heart cavity region and the classification method can detect heart abnormalities.

\section{Acknowledgements}

The authors would like to thank the Ministry of Research Technology and High Education and the Indonesia government for funding this research through research grant and also would like to thank the Politeknik Elektronika Negeri Surabaya.

\section{REFERENCES}

[1] J. A. Noble and D. Boukerroui, Ultrasound image segmentation: a survey,IEEE Transactions onMedical Imaging,vol. 25, pp. 987-1010, 2006.

[2] C. Petitjean and J.-N. Dacher, A review of segmentation methods in short axis cardiac MR images, Medical Image Analysis, vol. 15, pp. 169184, 2011.

[3] L. Hae-Yeoun, N. C. F. Codella, M. D. Cham, J. W. Weinsaft, and W. Yi, Automatic Left Ventricle Segmentation Using Iterative Thresholding and an Active Contour Model With Adaptation on Short-Axis Cardiac MRI, IEEE Transactions on Biomedical Engineering, vol. 57, pp. 905-913, 2010.

[4] J. W. Klingler, Jr., C. L. Vaughan, T. D. Fraker, Jr., and L. T. Andrews, Segmentation of echocardiographic images using mathematical morphology, IEEE Transactions onBiomedical Engineering, vol. 35, pp. 925-934, 1988.

[5] S. G. Lacerda, A. F. da Rocha, D. F. Vasconcelos, J. L. A. de Carvalho, I. G. Sene, and J. F. Camapum, Left ventricle segmentation in echocardiography using a radial-search-based image processing algorithm, Medicine and Biology Societyin Engineering, EMBS 2008. 30th Annual International Conference of the IEEE, pp. 222-225, 2008.

[6] M. d. C. dos Reis, A. F. da Rocha, D. F. Vasconcelos, B. L. M. Espinoza, F. A. d. O. Nascimento, J. L. A. de Carvalho, S. Salomoni, and J. F. Camapum, Semi-automatic detection of the left ventricular border, Medicine and Biology Societyin Engineering,EMBS 2008. 30th Annual International Conference of the IEEE, pp. 218-221, 2008.

[7] W. Ohyama, T. Wakabayashi, F. Kimura, S. Tsuruoka, and K. Sekioka, Automatic left ventricular endocardium detection in echocardiograms based on ternary thresholding method, Proceedings 15th International Conference onPattern Recognition, pp. 320-323 vol.4, 2000. 
[8] C. Jierong, F. Say Wei, and S. M. Krishnan, Watershed-presegmented snake for boundary detection and tracking of left ventricle in echocardiographic images, IEEE Transactions onInformation Technology in Biomedicine,vol. 10, pp. 414-416, 2006.

[9] S. G. Moursi and M. R. El-Sakka, Initial Contour for Ultrasound Carotid Artery Snakes, IEEE International Symposium onSignal Processing and Information Technology, pp. 390-395, 2007.

[10] S. Ranganath, Contour extraction from cardiac MRI studies using snakes, IEEE Transactions onMedical Imaging,vol. 14, pp. 328-338, 1995.

[11] V. Chalana, D. T. Linker, D. R. Haynor, and K. Yongmin, A multiple active contour model for cardiac boundary detection on echocardiographic sequences, IEEE Transactions onMedical Imaging,vol. 15, pp. 290-298, 1996.

[12] A. Laine and Z. Xuli, Border identification of echocardiograms via multiscale edge detection and shape modeling, Proceedings International Conference onImage Processing, 1996, pp. 287-290 vol.3, 1996.

[13] R. Sigit, M. M. Mustafa, A. Hussain, O. Maskon, and I. F. Nor, On the use of collinear and triangle equation for automatic segmentation and boundary detection of cardiac cavity images, Adv Exp Med Biol, vol. 696, pp. 481-8, 2011.

[14] M. Kass, A. Witkin, and D. Terzopoulos, Snakes: Active contour models, International Journal of Computer Vision, vol. 1, pp. 321-331, 1988.

[15] X. Chenyang and J. L. Prince, Snakes, shapes, and gradient vector flow, IEEE Transactions onImage Processing,vol. 7, pp. 359-369, 1998.

[16] Y. Itai, H. Kim, and S. Ishikawa, A segmentation method of lung areas by using snakes and automatic detection of abnormal shadow on the areas, International Journal of Innovative Computing, Information and Control, vol. 3, pp. 277-284, 2007.

[17] M. A. Jaffar, A. Hussain, A. M. Mirza, and A. u. Ch, Fuzzy Entropy and Morphology based fully automated Segmentation of Lungs from CT Scan Images, International Journal of Innovative Computing, Information and Control vol. 5, pp. 4993-5002, 2009.

[18] R. Sigit, M. M. Mustafa, A. Hussain, O. Maskon, and I. F. Mohd Nor, Cardiac Cavity Segmentation in Echocardiography using Search Contour and Snakes, in Industrial Electronics Seminar 2009, Surabaya, Indonesia, pp. C12-16, 2009. 